$8-2020$

\title{
A different strategy for management of a case of adenoid cystic carcinoma of bartholin gland
}

Rozilla Sadia Khan

Huda Saleem Abbasi

Follow this and additional works at: https://ecommons.aku.edu/

pakistan_fhs_mc_women_childhealth_obstet_gynaecol

Part of the Obstetrics and Gynecology Commons 
CASE REPORT

\section{A different strategy for management of a case of adenoid cystic carcinoma of Bartholin gland}

Rozilla Sadia Khan, Huda Saleem Abbasi

\begin{abstract}
Adenoid cystic carcinoma of the Bartholin gland is a rare vulval cancer. Available literature suggests an aggressive nature with protracted clinical symptoms and local recurrence despite adequate surgical excision with or without adjuvant radiotherapy. This case demonstrates the role of neoadjuvant chemotherapy and interstitial brachytherapy with less radical surgery, in the treatment of adenoid cystic carcinoma of the Bartholin gland. A 63year-old woman presented in the clinic four months after the removal of vulvar mass with histopathology report showing adenoid cystic carcinoma of the Bartholin gland with positive margins. Clinical examination and imaging showed local disease extension up to the levator ani and abutting anal margin. She was given four cycles of neoadjuvant chemotherapy followed by ipsilateral hemivulvectomy with inguino-femoral lymphadenectomy followed by 25 cycles of external beam radiation and three cycles of interstitial brachytherapy. The patient has been disease free for more than 30 months. The use of neoadjuvant chemotherapy in treatment of Bartholin carcinoma along with conservative surgery and radiotherapy can be a good treatment strategy as it reduces the morbidity associated with radical surgery without recurrence to date.
\end{abstract}

Keywords: Bartholin gland carcinoma, genital tract carcinoma, Adeno cystic carcinoma.

https://doi.org/10.5455/JPMA.29969

\section{Introduction}

Adenoid cystic Bartholin gland carcinoma (BG-ACC) is a very rarely reported carcinoma having an incidence of less than $1 \%$ of all female genital malignancies and $1-7 \%$ of all vulvar carcinomas. ${ }^{1}$ Available literature suggests an aggressive nature with protracted clinical symptoms and local recurrence despite adequate surgical excision with or without adjuvant radiotherapy. ${ }^{2}$ There is no consensus

Aga Khan University Hospital, Karachi, Pakistan.

Correspondence: Rozilla Sadia Khan. e-mail: rozilla.khan@aku.edu

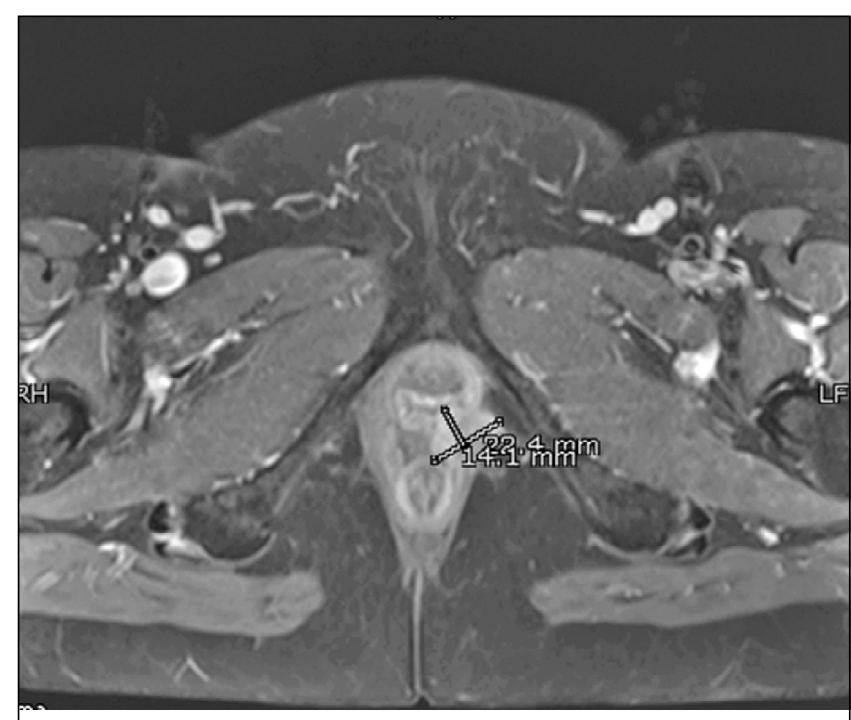

Figure: MRI - Bartholin gland ACC, axial view.

regarding the treatment of this carcinoma. This case demonstrates the role of neoadjuvant chemotherapy in the treatment of primary Bartholin cancer, reducing the need for radical vulvectomy.

\section{Case Report}

A 68-year-old lady para 2, menopausal for 18 years, presented with a vulval lump that had developed after excisional biopsy, at the Aga Khan University Hospital, Pakistan, in June 2016. She initially noticed a swelling four months back which was painful and had visited her doctor multiple times for urinary burning and vulval swelling and was given antibiotics with no resolution of symptoms. An excisional biopsy of the mass was done, but she delayed visiting her doctor postoperatively. On recurrence of the swelling, she self-referred to the hospital where histopathological slides were reviewed which confirmed the diagnosis of adenoid cystic carcinoma (ACC) of Bartholin gland with perineural invasion and margins positive for tumour. She had a family history of colorectal carcinoma as her sister too had the problem. The patient had not undergone any routine check-ups or prior

Vol. 70, No. 8, August 2020 
screening.

Clinical examination revealed a mass of $3 \times 2 \mathrm{~cm}$ that was partially mobile, hard, non-fluctuant and painful on palpation at the site of left labia minora. This mass was in close proximity to the perineal body and anus. Naked eye examination showed normal vaginal mucosa around the vaginal introitus. Per speculum examination did not reveal any abnormality in uterine cervix and vagina. There was no inguinal lymphadenopathy on examination.

Radiological examination, haematological, biochemical test and cervical cytology:MRI showed ill-defined infiltrating abnormal intensity area measuring $3.1 \times 1.4 \mathrm{~cm}$ which was found to have involved the posterior fornix and left lateral wall of the vagina. It was abutting the perineal body and infiltrating into left sided ischiorectal fossa with involvement of levator sling. A few subcentimeter pelvic and inguinal lymph nodes were noted. Cervical cytology was negative for intraepithelial lesion or malignancy. All routine haematological and biochemical tests were normal.

In view of the close proximity to the anal orifice, the need of radical vulvectomy with anal excisional procedure and colostomy was discussed with the patient who opted for neoadjuvant treatment, and was given four cycles of chemotherapy with Cylophosphamide, Adriamycin and Cisplatin. Follow up MRI of the pelvis showed interval decrease up to $1 \mathrm{~cm}$ of the previously seen lesion. After four cycles of chemotherapy and optimisation, the patient underwent left hemivulvectomy and left inguinofemoral lymphadenectomy. Biopsy of the specimen showed a tumour of $1.5 \times 1 \mathrm{~cm}$; the tumour was found $2 \mathrm{~cm}$ away from labia majora and $1.1 \mathrm{~cm}$ away from deep margins. Medial wall of the vagina and all lymph nodes were found tumour-free. After one month of surgery the patient received 25 cycles of $4500 c G y$ of external beam radiotherapy and four fractions of 12 Gray interstitial brachytherapy. After the treatment, the patient was followed up at the clinic with three-monthly clinical examination, six-monthly MRI for first year and then yearly. For 30 months since the treatment to date, there have been no signs of recurrence.

\section{Discussion}

ACC, first recognised in 1853 , is a specific variant of adenocarcinoma of the salivary and mucous gland. ${ }^{1}$ It is found in various locations such as salivary glands, breast, skin and lungs. ${ }^{1}$ BG-ACC is a slow growing tumour with a tendency for perineural invasion. The perineural invasion is characteristic and a possible cause for the pruritus and pain. The average age of patients is 47 years and the most common symptom is a painful nodule according to the 64 cases reported so far. ${ }^{2}$ It has been suggested that the possibility of cancer should be considered when women over the age of 40 present with a Bartholin's gland enlargement. ${ }^{2}$ Often, there is delay due to initial misdiagnosis as Bartholin's cyst, inadequately treated by drainage or marsupialisation. Enlargement of Bartholin's gland in women older than 40 years old should be considered malignant until proven otherwise.

There is no consensus regarding the optimal treatment. In a study by Yang et al radical vulvectomy and simple excision with or without inguino-femoral lymphadenectomy were done. Negative resection margin was achieved in $70 \%$ by radical vulvectomy versus $52 \%$ by simple excision. ${ }^{1}$ The need for inguinofemoral and pelvic lymph node dissection should not be considered unless the nodes are clinically and radiologically involved. If performed, it should at least be limited to the ipsilateral side of the tumour. ${ }^{3-6}$

While a primary surgical approach is recommended where resection is feasible, in some cases, complete resection may compromise midline structures such as the anus, clitoris, urethra and vagina resulting in an excessively mutilating and morbid surgical approach. Neoadjuvant chemotherapy or in combination with radiotherapy can avoid radical surgical complications for the patients. The goal of neoadjuvant treatment is to reduce the size of the tumour for radiotherapy or surgery, transform the inoperable tumours into radically resectable ones, avoid pelvic exenteration and assure a better quality of life. Furthermore, neoadjuvant treatments cure micrometastatic dissemination of the disease preventing a significant rate of relapse. ${ }^{7}$ In a study by Mousa et al neoadjuvant chemoradiation was followed by surgical excision of a BCG adenocystic carcinoma and the patient has been in remission for three years. ${ }^{8}$

Adjuvant radiotherapy is recommended if the surgical resection margins are positive or perineural invasion is identified. Local recurrence rate was $9.5 \%$ after adjuvant radiotherapy as against $37.5 \%$ for those who did not receive adjuvant radiation. ${ }^{9}$

In the present case, the tumour was in close proximity to the anus, which precludes achieving negative resection 
margins without posterior exenteration. To avoid complications and psychosexual impact associated with radical surgery, the options were discussed with the patient who opted for neoadjuvant chemotherapy with Cylophosphamide, Adriamycin and Cisplatin followed by surgical excision. In order to avoid adverse tissue effects and promote healing radiotherapy for local control was carried out post operatively. To our knowledge, this is the first case of this regimen of treatment.

\section{Conclusion}

In conclusion, primary carcinoma of Bartholin's gland is a very rare malignancy of the vulva. From this case we learnt that index of suspicion should be very high in women developing labial mass over 40 years of age, while treatment should be multimodal with exploration of new modalities to reduce morbidity and recurrence.

Disclaimer: None

Conflict of interest: None

Funding disclosure: None

\section{References}

1. Yang SYV, Lee JW, Kim WS, Jung KL, Lee SJ, Lee JH, et al. Adenoid cystic carcinoma of the Bartholin's gland: Report of two cases and review of the literature. Gynecol Oncol. 2006; 100:422-5.

2. Hung FY, Wang KL, Wang TY. Adenoid Cystic Carcinoma of Bartholin's Gland: A Case Report. Taiwan J Obstet Gynecol. 2005; 44:72-4.

3. Anaf V, Buxant F, Rodesch F, Simon P, Van de Stadt J, Noel JC, et al Adenoid cystic carcinoma of Bartholin's gland: what is the optimal approach? Eur J Surg Oncol. 1999; 25:406-9.

4. Webb J, Lott M, O'SULLIVAN J, Azzopardi J. Combined adenoid cystic and squamous carcinoma of Bartholin's gland. Case report. Br J Obstet Gynaecol. 1984; 91:291-5.

5. Lelle R, Davis K, Roberts J. Adenoid cystic carcinoma of the Bartholin's gland: the University of Michigan experience. Int J Gynecol Cancer. 1994; 4:145-9.

6. Abrao FS, Marques AF, Marziona F, Abrao MS, Junqueira LCU, Torlon $\mathrm{H}$. Adenoid cystic carcinoma of Bartholin's gland: review of the literature and report of two cases. J Surg Oncol. 1985; 30:132-7.

7. Ouldamer L, Chraibi Z, Arbion F, Barillot I, Body G. Bartholin's gland carcinoma: Epidemiology and therapeutic management. Surg Oncol. 2013; 22:117-22.

8. Mousa A, Rahimi K, Warkus T. Neoadjuvant chemoradiotherapy followed by radical vulvectomy for adenoid cystic carcinoma of Bartholin's gland: a case report and review of the literature. Eur J Gynaecol Oncol. 2016; 37:113-6.

9. Alsan C, Vinh-Hung V, Eren F, Abacio?lu U. Adenoid cystic carcinoma of the Bartholin's gland: case report and systematic review of the literature. Eur J Gynaecol Oncol. 2011; 32:567-72. 